\title{
Barriers to the use of a diagnostic oral microbiology laboratory by general dental practitioners
}

\author{
K. M. Roy, ${ }^{1}$ A. Smith, ${ }^{1}$ J. Sanderson, ${ }^{2}$ J. Bagg, ${ }^{1}$ D. MacKenzie, ${ }^{1}$ M. S. Jackson, ${ }^{1}$ \\ G. Taylor, ${ }^{3}$ and J. McEwen, ${ }^{4}$
}

\begin{abstract}
Objective To identify barriers on the use of diagnostic microbiology facilities in general dental practice.

Design A cross-sectional survey using a postal questionnaire. Setting Primary/secondary care interface between the diagnostic oral microbiology laboratory, University of Glasgow Dental Hospital and School, Glasgow and dental practitioners within the surrounding health boards, 1998.
\end{abstract}

Subjects All GDPs (797) within Argyll and Clyde, Ayrshire and Arran, Lanarkshire and Greater Glasgow Health Boards.

Main outcome measures The responses were expressed as both absolute and relative frequencies.

Results Responses were received from 430 (55\%). The most frequent reason for failure to use the service was lack of information, with more than half of the respondents claiming to be unaware of the facility. Lack of request forms and sampling equipment were also viewed as barriers to using the service. Conclusions The laboratory is failing to successfully communicate its role in addressing the growing burden of antibiotic resistance in the community and must be more proactive in encouraging appropriate use and increasing accessibility of the service to GDPs.

In 1997, the Select Committee on Science and Technology of the House of Lords carried out a public inquiry into the increasingly pressing problem of the development of antibiotic resistance. Among others, the report recommended the more prudent use of antibiotics in human medicine supported by a number of surveillance and education measures. ${ }^{1}$

Within the UK, prescriptions of antibiotics by the dental profession cost the General Dental Services at least $£ 4.5$ million per year. ${ }^{2}$ On top of such visible sums are the potential hidden expenses caused by the treatment of antibiotic resistant infections. The contribution of the dental profession to the worldwide problem of antibiotic resistance remains as yet unknown.

The prescription of antibiotics is an important adjunct to the practice of dentistry, playing an important role both in the management of suppurative infections such as dento-alveolar abscesses and acute periocoronitis, and in prophylactic cover of medically-compromised patients. As in general medicine, this privilege is routinely abused and it has been shown that prescribing is generally suboptimal with wide variations both in the types and doses of antibiotics. ${ }^{3,4}$ Furthermore,

${ }^{1}$ Infection Research Group, University of Glasgow Dental School, 378 Sauchiehall Street, Glasgow G2 3JZ2 Audit Facilitator, Glasgow Dental Hospital NHS Trust, 378 Sauchiehall Street, Glasgow G2 3JZ ${ }^{3}$ General Dental Practitioner, Woodside Health Centre, Barr Street, Glasgow G20 7LR ${ }^{4}$ Department of Public Health, University of Glasgow, 2 Lilybank Gardens, Glasgow G12 8RZ

REFEREED PAPER

Received 11.09.98; accepted 07.01.99

(C) British Dental Journal 1999; 186: 345-347 most prescribing is empirical with broad-spectrum agents being used increasingly. This creates two important problems. First, it encourages resistance to a wide range of agents and decreases options for prescribing in the future. Secondly, it eradicates more of the normal protective flora, rendering patients more susceptible to super-infection by resistant pathogenic strains. Recent studies have provided evidence of an increase in the levels of antibiotic resistance among organisms isolated from the oral cavity. ${ }^{5-9}$ Furthermore, oral streptococci, such as $S$. oralis, have been implicated as a source of the penicillin resistance trait in S. pneumonia. ${ }^{10}$

Since antimicrobial mis-use and over-use is a major risk factor for the development of resistance, ${ }^{11}$ improved prescribing must be a cornerstone to tackling resistant organisms. Crucial to this process is involvement of the clinical microbiology laboratory, which not only provides the data on which therapeutic decisions that discourage the development of resistance can be made, but also provides the expertise needed for resistance surveillance. Further, this information is useful in the development of local policies and guidelines on antibiotic prescribing, the routine dissemination of which have an important educational value and beneficially influence prescribing habits. ${ }^{12}$

Encouraging GDPs to prescribe rationally is a highly desirable objective. However, the role of the diagnostic microbiology laboratory in its achievement has usually been by default, generally being consulted for common infections only when empirical therapy has failed. In an attempt to encourage GDPs to use the diagnostic oral microbiology laboratory at the Glasgow Dental Hospital and School as an aid in their decision-making process for antibiotic treatment, this study took a consultative approach to determine their opinions on the current service. Information was also sought regarding potential barriers to its use.

\section{Methods and materials}

All GDPs $(1,864)$, in every health board within Scotland have access to the facilities provided by the Glasgow Dental Hospital. However, in reality, GDPs practising within the Western and Central health boards are more likely to use it. The extent of the problem was analysed by examining laboratory records from 1993 to 1997. The total number of specimens received from GDPs ranged from 45 to 17 per year, with a $40 \%$ reduction being observed during the 5 -year period. This suggested that prescribing in general dental practice was empirical. Examining samples collected from dento-alveolar abscesses provided an indication of the quality of the specimens submitted. Of the 40 specimens received, seven (18\%) were considered an appropriate specimen, that is, an aspirate of pus. The remainder comprised pus swabs or paper point specimens.

Following piloting, a questionnaire together with a personalised letter and a pre-paid reply envelope were mailed to all dentists (797) practising within the four health boards surrounding the Glasgow Dental Hospital, namely Argyll and Clyde, Ayrshire and Arran, Lanarkshire and Greater Glasgow Health Board. Non- 
respondents were sent a further explanatory letter three weeks after the initial mailing.

The questionnaire contained 18 items. A combination of closeended questions (both ordered and unordered) and partially closed questions were posed. Care was taken with the order of the questions, not to make the questions repetitive, and to make the questionnaire aesthetically pleasing and easy to complete. The overall design of the questionnaire aimed to encourage the response through three mechanisms. First by using a consultative approach, giving the true impression that the views and opinions of the dentists were appreciated. Secondly, by ensuring that the physical and mental effort required to complete the questionnaire was minimal and thirdly, the researchers sought to establish trust by identifying with a known organisation that has legitimacy.

The questionnaire was developed in three parts. Part A collected information to explore how the demographics of the responding GDPs who used the service differed from those who did not. Data on gender, practice distance from Glasgow Dental Hospital, practice size and practice speciality were collected. Part B was designed to assess the attitudes of the GDPs who had used the service previously. Views were sought on the request forms, the transportation and processing of specimens, reporting procedures, microbiological advice provided, and the quality of the service as a whole. Information was also obtained on the frequency of use of the service. The final section sought opinions on a number of potential barriers to the service. GDPs were also asked about their views on a number of potential services being considered by the laboratory to improve the use of the facility. Information was also collected on the attitudes of the GDPs toward microbiological sampling for a number of clinical conditions and for monitoring antibiotic sensitivity levels. The final questions in this section clarified whether GDPs were using alternative facilities and whether they were aware that a fee was payable by the NHS for each sample submitted for microbiological examination.

Non-bias response was assessed in terms of the variables that were known for the entire sample being studied, namely distance from Glasgow Dental Hospital as defined by the health board and practice size. As non-respondents are considered to be more like late respondents than early respondents, ${ }^{13}$ the representativeness of the respondents was determined by comparing early respondents (those received following the initial mailshot) with late respondents (those received after the explanatory letter), as well as respondents with non-respondents.

Data analysis was carried out using Minitab. Frequencies were used to examine the distribution of responses for all the variables and to describe sample demographics. The association between variables was examined by cross-tabulations and the statistical significance of such relationships examined by chi-squared analysis.

\section{Results}

Twenty of the 797 questionnaires were returned as undelivered and it was assumed that 777 questionnaires had reached their destination; 430 completed questionnaires were returned representing a 55\% response rate discounting undelivered questionnaires.

There was no evidence of non-response bias when comparing early respondents with late respondents. Comparison of all respondents with non-respondents also indicated no difference with regard to the distance from the laboratory. However, there was a significant difference between practice size and whether or not the GDPs responded. In particular, the number of GDPs practising single-handed was under-represented.

Of the responding GDPs, just under half (48\%) were aware of the diagnostic microbiology service. However, only 68 (15\%) of the respondents had previously used it. There were no significant differences between those dentists who had used the service and those who had not with regard to gender, practice distance from the laboratory, practice size and speciality. An alternative laboratory had been consulted by 33 (7.7\%) of the responding GDPs. Although the
Table 1 Barriers to the use of the oral microbiology service by previous users and non-users

\begin{tabular}{lccc}
\hline Barrier & $\begin{array}{c}\text { Previously used the microbiology service } \\
\text { Yes }(N=63)\end{array}$ & Po - value \\
& & & \\
& 12 & 288 & \\
Lack of information & 6 & 31 & $<0.001$ \\
Time collecting samples & 8 & 29 & 0.78 \\
Time taken to get result & 14 & 135 & 0.21 \\
Lack of request forms & 16 & 78 & 0.025 \\
Lack of address labels & 19 & 162 & 0.46 \\
Lack of equipment & 5 & 17 & 0.037 \\
Cost & 13 & 92 & 0.45 \\
Problems with transportation & & &
\end{tabular}

use of a local hospital was more common by GDPs practising further away, the difference was not statistically significant.

Respondents who had previously used the microbiology service were asked to express their degree of satisfaction with the service by choosing one of the following options: excellent, good, average or poor. Opinions were sought on the respondent's experience with the request forms, the transportation procedures, turnaround time, the format and delivery of the report, microbiological advice provided and the quality of the service as a whole. The data indicated a high degree of satisfaction with the service although around a quarter failed to comment on each issue. This may be indicative of the practitioner being unable to recall the details of any interaction in the past.

From a list provided, respondents were asked to identify the barriers to their use of the service. This included an 'any other' option that invited the dentist to comment specifically on any obstacle that they felt existed which had not been already covered. Of those proposed, lack of information about the service was considered the biggest problem by $300(70 \%)$. This was followed by lack of sampling equipment $(181 ; 42.1 \%)$, lack of request forms $(149 ; 34.6 \%)$ and issues concerning transport $(105 ; 24.4 \%)$. Time taken to collect the samples and the time taken to receive the results were seen equally as a barrier by $37(8.6 \%)$. Lack of address labels was considered a barrier by $94(21.9 \%)$ and cost by $22(5.1 \%)$. Fifteen respondents felt there were barriers other than those listed including:

- Feeling that microbiological sampling was not necessary

- Distance.

Cross-tabulation followed by chi-squared analysis showed a statistically-significant relationship between not using the service and lack of information, sampling equipment and request forms (Table 1).

Of the proposed solutions to these barriers, 365 (84.9\%) of the respondents felt an information booklet describing the service would be of use. This was closely followed by provision of sampling packs containing the necessary equipment for specimen collection $(81.6 \%)$. Information on taking samples and the transport of specimens was also considered appropriate by a similar number $(67.7 \%)$.

The attitudes toward microbial sampling in general practice indicated that with the exception of determining antibiotic sensitivities, microbiological sampling was not considered important for monitoring endodontic treatment, periodontal treatment, implants, dental abscesses, denture stomatitis or pericoronitis. There was, however, a wide variation in opinions expressed.

\section{Discussion}

In view of the changing epidemiology of antibiotic resistance in the community, we felt it prudent to examine the role of a specialist oral microbiology diagnostic service available to GDPs and the barriers to the use of such a service. We were encouraged to find that a large proportion of GDPs were supportive of the role of the laboratory but concerned to find that the majority were unaware of it. One could argue that the high percentage of non-respondents suggests an even greater lack of interest in the service than indicated here. This argument hinges on the effect of non-response bias, which does increase 


\section{RESEARCH oral medicine}

when the response rate is low and when non-respondents differ from respondents in the parameters of interest. ${ }^{13}$ There was, however, no evidence of non-response bias with the exception that the opinions of GDPs practising alone may be under-represented. Thus, although the response rate of the recipients was in the suspect category as specified in the $B D J,{ }^{14}$ it was felt that the results provided a good indication of the potential barriers which prevent the use of the diagnostic service for bacteriological identification and antibiotic sensitivity testing.

Lack of time, both for collecting samples and obtaining the laboratory results was hypothesised initially as being an important variable preventing the use of the diagnostic service to aid rational prescribing. This was not, however, shown to be significantly associated with the lack of use and neither was it considered an impediment by the respondents. Issues regarding time have encouraged the development of 'rapid tests.' ${ }^{1}$ Yet even with a faster turnaround time, the results are not available at the time antimicrobials are prescribed. It may be that the respondents felt that improving turnaround time would not affect how they prescribed in practice. This is where knowledge of local antibiotic sensitivity profiles can aid the initial decision of which antibiotic to prescribe.

Lack of time for collecting a specimen is more likely to be an issue with dentists practising single-handed and, as mentioned, this group of practitioners was under-represented. Therefore, the effect of this variable on the use of the diagnostic facility remains unanswered from this survey.

The effect of age was not investigated in this study as demographic information available on GDPs who had used the service previously indicated that the pressures of time and distance from the service may overrule any effect of age. However, age (or year of qualification), may be important and should be considered in future studies.

The majority of respondents considered lack of information about the service as the biggest barrier to its use. This study identified that a major need of the GDPs was access to more comprehensive information on the service provided. The provision of a laboratory handbook containing information on the range of tests available, on specimen collection procedures and on recommended transportation requirements, is an obvious way of meeting this need and was considered to be constructive by the majority of practitioners who responded to the survey. Such information should be readily available from all laboratories with clinical pathology accreditation (CPA) status. However, it seems unlikely that provision of this information alone would change current behaviour and it must be followed through with continuing professional education and development. GDPs receive a fee of $£ 7.90$ from the NHS for microbiological sampling but few appear to know about it and those that do feel it is somewhat inadequate.

It was reassuring that more than three-quarters of the respondents appreciated the need for development of antibiotic guidelines and the provision of regular updates on local antibiotic sensitivity profiles. This indicates that GDPs are amenable to advice on antimicrobial management of their patients and that rational prescribing could be achieved in the primary dental care setting. It was also reassuring that GDPs were aware of the importance of microbial sampling for this purpose, though only $15 \%$ claimed to have previously obtained a specimen and sent it to the laboratory for bacteriological identification and sensitivity testing.

Dentists appear to be unaware of how or where the information on local sensitivities, used for the development of guidelines and policies is obtained. This is reflected by the fact that most considered microbiological sampling to be of only average importance in endodontic and periodontal treatment, for monitoring implant success, and for the management of dental abscesses, denture stomatitis and pericoronitis. All are common scenarios encountered routinely in general practice resulting in (often inappropriate) prescription of antimicrobial agents. There is a gap in the knowledge of dentists regarding the importance to the clinical laboratory of samples collected in general practice for microbiological analysis, without which surveillance of resistance cannot be undertaken and appropriate policies developed; this message must be communicated. However, it needs to be one that emphasises the need for appropriate clinical specimens. Misleading or incorrect laboratory reports invite inappropriate prescribing, particularly if contaminating commensals are reported as significant.

The only way to remove the uncertainty in prescribing is to examine clinical material bacteriologically to identify the infecting organism and its antibiotic sensitivity profile. However, we do not recommend that GDPs establish antimicrobial susceptibility before every antibiotic prescribed. This would at present be impracticable and financially impossible in today's climate. The BDA advises that bacteriological sampling should always be carried out in severe infections. Yet this survey indicates that the vast majority of dentists fail to do so. Guidelines on the appropriate use of the microbiology laboratory are therefore needed to complement policies on preventing antimicrobial resistance.

\section{Conclusion}

The microbiology laboratory has an important role to play in monitoring the emergence of antibiotic resistance in oral infections and to slow down the spread of resistant strains. Specifically, these objectives can be met through active surveillance and the promotion of rational and moderate antibiotic use. Each affects and depends on the other and cannot be performed without the help of the GDPs in the community setting. This survey has indicated that GDPs are aware of the importance of microbial sampling for monitoring resistance but their failure to do so, in this situation, resulted from a lack of communication. Diagnostic laboratories must take a proactive role in encouraging appropriate use and making their facilities as accessible as possible to the GDPs. This will permit accurate and rational prescribing and allow the profession to cooperate with other health care professionals in addressing the growing burden of antibiotic resistance in both hospitals and the community.

1 Select Committee on Science and Technology. Resistance to antibiotics and other antimicrobial agents. London: HMSO, 1998.

2 Dental Practice Board. Digest of statistics, 1996/97, Part 1: Detailed analysis of GDS treatment items. Eastbourne: Dental Practice Board, 1998.

3 Lewis M A O, Meechan C M, MacFarlane T W, Lamey P J, Kay E J. Presentation and antimicrobial treatment of acute orofacial infections in general dental practice. Br Dent J 1989; 166: 41-45.

4 Thomas D W, Satterthwaite J, Absi E G, Shepherd J P. Antibiotic prescription for acute dental conditions in the primary-care setting. $\mathrm{Br}$ Dent J 1996; 181: 401-404.

5 Heimdahl A, von Konow L, Nord C E. Beta lactamase producing Bacteroides species in the oral cavity in relation to penicillin therapy. $J$ Antimicrob Chemother 1981; 8: 225-229.

6 Teng L-J, Hsueh P-R, Chen Y-C, Ho S-W, Luh K-T. Antimicrobial susceptibility of viridans group streptococci in Taiwan with an emphasis on the high rates of resistance to penicillin and macrolides in Streptococcus oralis. J Antimicrob Chemother 1998; 41: 621-627.

7 Olsvik B, Hansen B F, Tenover F C, Olsen I. Tetracycline-resistant microorganisms recovered from patients with refractory periodontal disease. J Clin Periodont 1995; 22: 391-396.

8 Kononen E, Saarela M, Kanervo A, Karjalainen J, Asikainen S, Jousimiessomer H. Beta-lactamase production and penicillin susceptibility among different ribotypes of prevotella-melaninogenica simultaneously colonizing the oral cavity. Clin Infect Dis 1995; 20: S364-S366.

9 Lewis M A O, Pankhurst C L, Douglas C I et al. Prevalence of penicillinresistant bacteria in acute suppurative oral infection. J Antimicrob Chemother 1995; 36: 883.

10 Coffey T J, Dowson C G, Daniels M, Spratt B G. Horizontal spread of an altered penicillin-binding protein $2 \mathrm{~b}$ gene between streptococcus-pneumoniae and streptococcus-oralis. Fems Microbiol Letters 1993; 110: 335-339.

11 Greenwood D. Tarnished gold - 60 years of antimicrobial drug-use and misuse. J Med Micro 1995; 43: 395-396.

12 Sahm D F. The role of clinical microbiology in the control and surveillance of antimicrobial resistance. ASM News 1996; 62: 25-29.

13 Siemiatycki J, Campbell S. Nonresponse bias and early versus all responders in mail and telephone surveys. Am J Epidemiol 1984; 120: 291-301.

14 Rugg-Gunn A. Guidelines for acceptable response rates in epidemiological surveys. Br Dent J 1997; 182: 68.

15 British Dental Association, British Medical Association, and the Royal Pharmaceutical Society of Great Britain. Dental Practitioners' Formulary/ British National Formulary (1996-1998). London: British Medical Association and The Pharmaceutical Press, 1996. 